\title{
Relation entre température, teneur en glucides et respiration de la plante entière chez la tomate en phase végétative
}

\author{
Christian GARY \\ avec la collaboration technique de Béatrice BRLiNEL \\ I.N.R.A., Station de Bioclimatologie, Centre de Recherches d'Avignon, Domaine Saint-Paul, B.P.91, F 84140) \\ Montfavet
}

\begin{abstract}
On impose à de jeunes plants de tomate la mobilisation de leurs réserves glucidiques pendant une période d'obscurité prolongée de 48 heures et ce, à différents niveatux de température $\left(10,15,20\right.$ et $\left.25^{\circ} \mathrm{C}\right)$. Les activités respiratoires et les teneurs en glucides, mesurées périodiquement, sont mises en relation dans les parties aériennes et dans les racines. La fraction glucidique la plus limitante serait le saccharose. La respiration de la plante entière dépend de la quantité de substrat glucidique disponible (tous glucides confondus) suivant une relation hyperbolique. La température agit sur l'asymptote de cette courbe. La sensibilité de la tomate au froid se traduit par une rupture de pente de la relation d'Arrhenius entre respiration maximale et température vers $11{ }^{\circ} \mathrm{C}$. L'importance des besoins en énergie respiratoire pour l'entretien, la croissance et l'absorption des éléments minéraux modifie vraisemblablement la réponse de l'activité respiratoire à la teneur en glucides.
\end{abstract}

Mots clés additionnels: Contrôle de la respiration, sensibilité au froid, parties aériennes, racines, Lycopersicon esculentum Mill..

Relationship between temperature, carbohydrate content and respiration in the whole tomato plant during the vegetative phase.

Mobilization of stored sugars was induced in tomato seedlings by a 48-h night, and at 4 temperatures (10, 15, 20 and $25^{\circ} \mathrm{C}$ ). Respiration and carbohydrate content were measured at intervals on shoots and roots and their relationships studied. Sucrose seemed to be the most limiting sugar. Whole-plant respiration was related to the amount of available carbohydrate by a hyperbolic relationship. Sensitivity to chilling was observed around $11{ }^{\circ} \mathrm{C}$ with a change in slope of the Arrhenius relationship between maximum respiration and temperature. The response of respiration to carbohydrate content was likely modified by the respiratory energy demand for maintenance, growth and ion uptake.

Additional key words : Respiration control, sensitivity to chilling, shoots, roots, Lycopersicon esculentum Mill.

\section{INTRODUCTION}

De façon générale, dans la plupart des cellules végétales, les glucides constituent le principal substrat respiratoire (AP REES, 1980). La très forte dépendance de la respiration à l'égard de la teneur en glucides a été démontrée aussi bien à l'échelle de l'organe que de la plante entière. BARKER (1936) l'a mise en évidence sur des tubercules de pomme de terre, Saglio \& Pradet (1980) sur des pointes de racines de maïs aussitôt après la germination et FrosSARD (1985a) sur des systèmes racinaires entiers de jeunes plants de tournesol (entre 3 et 5 semaines). YeMm (1965) puis AzCón-Bieto \&
OSMOND (1983) l'ont décrite sur feuilles d'orge et de blé, Penning De VRies et al. (1979) et Coggeshall \& HoDGEs (1980) sur plantes entières (blé, maïs, ray-grass, soja). L'allure des courbes de réponse obtenues est en général assez conforme à la formulation théorique de THORNLEY (1972) qui propose une relation michaelienne entre activité respiratoire et teneur en glucides. Pourtant les modèles de bilan carboné à l'échelle de la plante entière ignorent cette relation ou la considèrent comme simplement linéaire et indépendante de la température (MC CREE, 1982).

La réponse de l'activité respiratoire à la température 
présente en général une forme exponentielle (YEMM, 1965), que l'on traduit par l'équation d'Arrhenius ou par l'équation du $Q_{10}$ (JOHNSON \& THORNLEY, 1985). Cependant l'effet de la température n'est pas identique en début et en fin de nuit au niveau de la feuille (AzCÓN-Bieto \& OSMOND, 1983) comme à celui du système racinaire (FROSSARD, 1985b): la quantité de substrat glucidique disponible est mise en cause, mais son rôle n'a pas été évalué jusqu'à présent de façon précise.

L'interaction entre les effets teneur en glucides et température sur l'activité respiratoire des parties aérienne et souterraine fait l'objet de l'expérience présentée ici. Chaque fraction glucidique est examinée séparément. Pour cela, activités respiratoires et teneurs en glucides sont mesurées simultanément tout au long d'une période d'obscurité prolongée (48 heures) et ce, à différents niveaux de température. Une expression simple de la respiration de la plante entière en fonction de la température et de la teneur en glucides est proposée, qui pourra être intégrée dans un modèle de bilan carboné.

\section{MATÉRIEL ET MÉTHODES}

\section{A. Matériel végétal}

Cet essai est réalisé sur de jeunes plants de tomate (variété Mélody) en phase végétative, 5 à 6 semaines après le semis, avant l'apparition du premier bouquet floral (8 à 10 feuilles apparentes). Ces plantes sont cultivées en chambre de culture dans les conditions climatiques suivantes (moyenne \pm écart maximum) :

jour : durée $=10$ heures,

température de l'air et de la solution nutritive $=$ $20 \pm 1{ }^{\circ} \mathrm{C}$

humidité relative $=60 \pm 5 \mathrm{p} .100$,

rayonnement $=210 \pm 20 \mu \mathrm{mol} \cdot \mathrm{m}^{2} \cdot \mathrm{s}^{\text {! }}$,

teneur en $\mathrm{CO}_{2}=400 \pm 20 \mathrm{ppm}$.

nuit: durée $=14$ heures,

température de l'air et de la solution nutritive = $15+1{ }^{\circ} \mathrm{C}$

humidité relative $=70 \pm 5 \mathrm{p} .100$

Leur état de croissance au moment de leur utilisation est le suivant (moyenne \pm écart maximum) :

poids sec des parties aériennes $=2,3 \pm 1,0 \mathrm{~g}$,

- poids sec des racines $=0,5 \pm 0,2 \mathrm{~g}$,

- surface foliaire $=0,06 \pm 0,02 \mathrm{~m}^{2}$,

- vitesse relative de croissance $=$

$0,16 \pm 0,01 \mathrm{~g}^{\prime} \mathrm{g}^{1} \cdot$ jour ${ }^{1}$.

Un semis est réalisé chaque semaine de façon à disposer en permanence de plantes au stade voulu. Etant donnée la vitesse relative de croissance, il suffit d'un délai de 3 jours pour que le poids sec total augmente de plus d'un gramme.

La solution nutritive, convenablement aérée, contient $3,5 \mathrm{mM}$ de $\mathrm{KNO}_{3}, 2,5 \mathrm{mM}$ de $\mathrm{Ca}\left(\mathrm{NO}_{3}\right)_{2}, 1,2 \mathrm{mM}$ de $\mathrm{MgSO}_{4}$ et $1,2 \mathrm{mM}$ de $\mathrm{NH}_{4} \mathrm{H}_{2} \mathrm{PO}_{4}$. Elle contient également les oligo-éléments nécessaires à la croissance:
23,0 $\mu \mathrm{M}$ de $\mathrm{H}_{3} \mathrm{BO}_{3}, 4,6 \mu \mathrm{M}$ de $\mathrm{MnCl}_{2}, 0,17 \mu \mathrm{M}$ de $\mathrm{CuSO}_{4}, 0,38 \mu \mathrm{M}$ de $\mathrm{ZnSO}_{4}, 0,05 \mu \mathrm{M}$ de $\left(\mathrm{NH}_{4}\right)_{6} \mathrm{Mo}_{7} \mathrm{O}_{24}$ et $19,8 \mu \mathrm{M}$ de Fe sous forme chélatée (EDDHA).

\section{B. Mesure des activités respiratoires et des teneurs en glucides}

Activités respiratoires et teneurs en glucides sont mesurées sur des plantes laissées à l'obscurité pendant 48 heures et donc mises en situation de mobiliser leurs réserves glucidiques. Ces mesures sont effectuées à 4 niveaux de température $\left(10,15,20\right.$ et $\left.25^{\circ} \mathrm{C}\right)$. Afin de préciser l'allure de la courbe de réponse de la respiration à la température, des mesures complémentaires d'activité respiratoire sont réalisées à $7{ }^{\circ} \mathrm{C}$. Le traitement température est imposé 24 heures avant le début de cette période d'obscurité prolongée afin de démarrer les mesures dans des conditions thermiques bien stabilisées (variations inférieures à $0,5^{\circ} \mathrm{C}$ de part et d'autre de la consigne). L'activité respiratoire des parties aérienne et souterraine est mesurée toutes les 10 minutes en chambre d'assimilation. Il y a 6 à 10 répétitions par traitement température. Les dosages de glucides sont réalisés sur des plantes placées en chambres de culture dans des conditions climatiques identiques. Après quelques mesures préliminaires de respiration, il est décidé d'effectuer les prélèvements comme suit : en début de nuit puis au bout de $14 \mathrm{~h}$ (durée normale de la nuit en chambre de culture), $24 \mathrm{~h}$ (après une diminution rapide d'activité respiratoire) et $41 \mathrm{~h}$ (quand la respiration s'est stabilisée). Chaque prélèvement se compose d'un lot de 3 plantes lyophilisées et broyées ensemble. Il y a 2 répétitions pour chaque traitement température.

Les mesures d'échanges de $\mathrm{CO}_{2}$ ont été réalisées dans des chambres d'assimilation fonctionnant en système ouvert (LONGUENESSE et al., 1982) et permettant de travailler sur parties aérienne et souterraine séparément. Une mesure en absolu de la teneur en $\mathrm{CO}_{2}$ est effectuée à l'entrée et à la sortie des 2 compartiments. Une correction est faite pour tenir compte de la dilution du $\mathrm{CO}_{2}$ par la vapeur d'eau libérée par la transpiration et de l'inertie liée au volume de l'enceinte (GARY, 1988). Le $\mathrm{pH}$ de la solution nutritive évolue peu du début à la fin de la manipulation : en général de 5,5 à 6,0. L'équilibre $\mathrm{CO}$, dissous-acide carbonique reste assez stablc. Le calcul montre que, dans cette gamme de $\mathrm{pH}$, les phénomènes de piégeage ou de libération parasite de $\mathrm{CO}_{2}$ sont négligeables par rapport à l'activité des racines. Les valeurs qui seront mises en relation avec les teneurs en glucides sont les moyennes des mesures de respiration réalisées pendant l'heure (6 mesures) au cours de laquelle les prélèvements pour les dosages sont réalisés.

Pour les dosages de glucides, l'extraction des glucides solubles est réalisée sur $50 \mathrm{mg}$ de poudre lyophilisée dans un mélange ternaire eau - méthanol - chloroforme avec adjonction d'une quantité connue de ribose, utilisé comme produit étalon. Une fraction du surnageant est prélevée et desséchée. Après lavage, le culot subit un empesage ( 1 heure à $100^{\circ} \mathrm{C}$ ) puis l'amidon est hydrolysé à l'amyloglucosidase $\left(18\right.$ heures à $\left.37^{\circ} \mathrm{C}\right)$ avec à nouveau adjonction de ribose. Une fraction de surnageant est prélevée et desséchée. Les 2 extraits ainsi obtenus subissent une silylation dans la pyridine anhydre (SWEELEY et al., 1963) avant de passer au chromatographe en phase 
gazeuse. Sont ainsi dosés dans le $1^{\text {er }}$ extrait, le fructose, le glucose et le saccharose et dans le $2^{c}$, l'amidon. Leurs teneurs seront exprimées en $\mathrm{g} \cdot \mathrm{g}{ }^{\prime}$ de matière sèche. Les glucides phosphates, qui sont les substrats immédiats de la glycolyse, n'apparaissent pas puisque les groupes acide phosphorique ne réagissent pas avec le réactif de silylation. L'amidon n'est pas dosé dans les racines car des essais préliminaires ont montré que, dans le matériel végétal utilisé, sa teneur ne dépasse pas des valeurs de l'ordre de notre seuil de détection soit $0,001 \mathrm{~g} \cdot \mathrm{g}^{1}$.

\section{RÉSULTATS}

\section{A. Evolution des activités respiratoires et des teneurs en glucides}

Les prélèvements effectués successivement au cours d'une période d'obscurité prolongée ont permis d'obtenir pour chaque traitement température une large

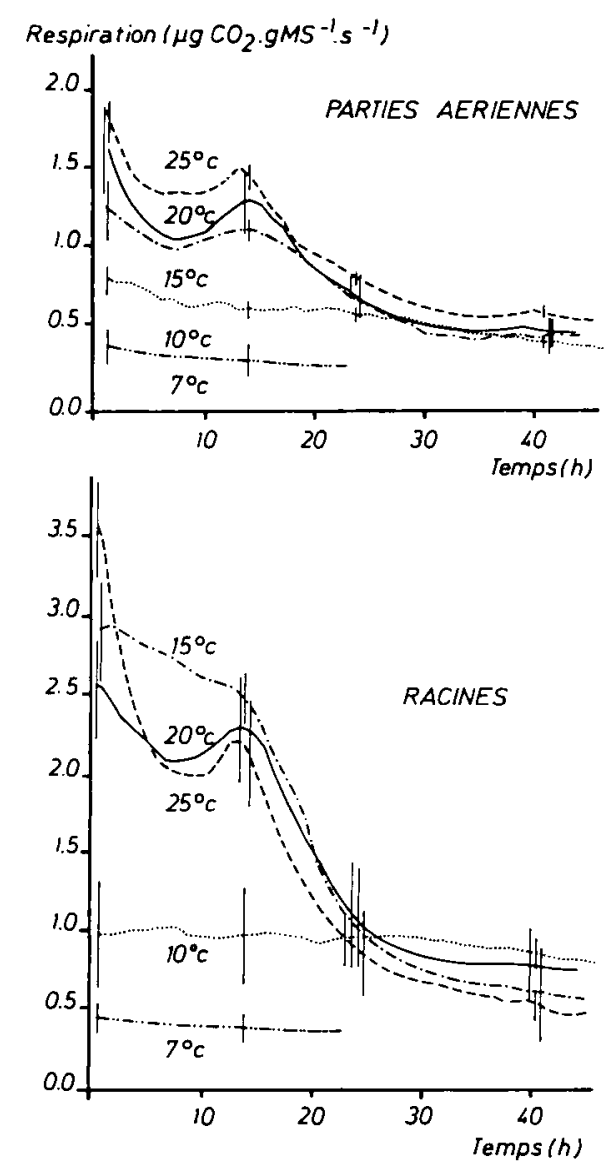

Figure 1

Evolution de lactivité respiratoire des parties aérienne et souterraine de jeunes plants de tomate soumis à différentes températures pendant une période d'obscurité prolongée de 48 heures. Afin que le graphique soit plus clair, les intervalles de confiance $(\alpha=0,10)$ sont représentés seulement aux moments des prélèvements pour les dosages de glucides. Ils ne seront pas repris dans les figures 3 à 6.

Shoot and root respiration of tomato seedlings versus time at different temperatures during a $48-h$ night. In order to get a clearer figure, confidence intervals $(\alpha=0.10)$ are only presented at the time of sugar analysis. They will not be mentioned again in figures 3 to 6 .
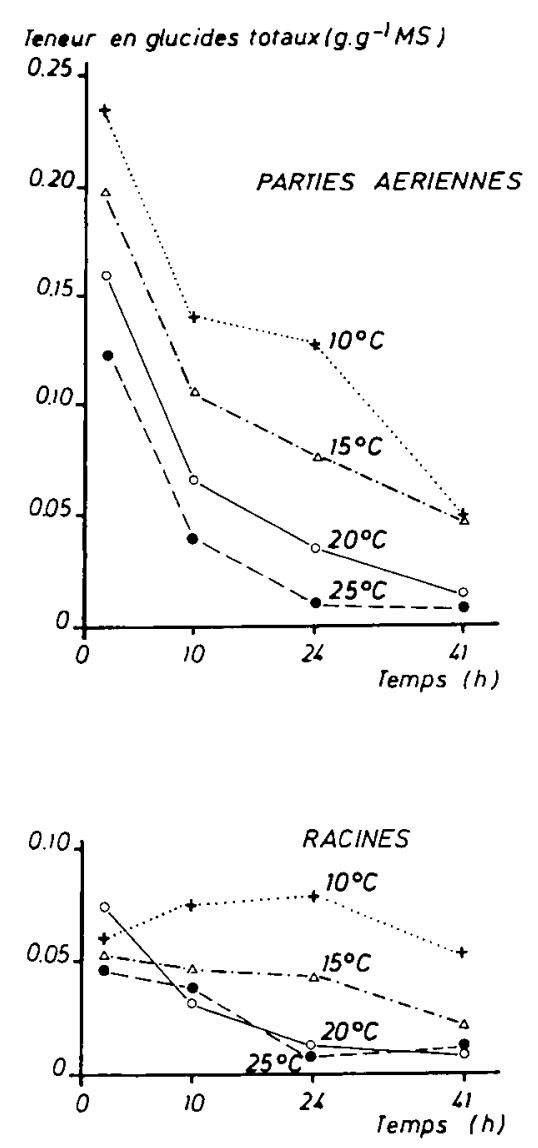

Figure 2

Evolution des teneurs en glucides des parties aérienne et souterraine de jeunes plants de tomate soumis à différentes températures pendant une période d'obscurité prolongée de 48 heures. Les valeurs représentées sont la moyenne de 2 répétitions.

Shoot and root carbohydrate content of tomato seedlings versus time at different temperatures during a 48 -h night. Each value is the mean of 2 replicates.

gamme de teneurs en glucides $\left(0,05\right.$ à $0,22 \mathrm{~g} \cdot \mathrm{g}{ }^{\prime}$ à $10^{\circ} \mathrm{C}, 0,02$ à $0,16 \mathrm{~g} \cdot \mathrm{g}{ }^{1}$ à $25^{\circ} \mathrm{C}$ ). L'activité respiratoire décroît puis reprend sensiblement pendant les premières 14 heures avant de décroître rapidement (fig. 1). A 7 et $10{ }^{\circ} \mathrm{C}$, ce type d'évolution n'apparaît pas; l'activité respiratoire baisse très lentement avec le temps. La respiration, ramenée à l'unité de masse de matière sèche, est nettement plus intense au niveau des racines qu'à celui des parties aériennes. Les teneurs en glucides totaux semblent décroître plus régulièrement mais le faible nombre de points ne permet pas de connaître leur évolution pendant les premières 14 heures (fig. 2). Les valeurs de départ ne sont pas identiques pour tous les traitements température puisque ceux-ci sont appliqués 24 heures avant le début des mesures. Il y a eu pendant ce temps accumulation de glucides dans les parties aériennes, d'autant plus que la température était basse. Cela traduit des activités respiratoires, des vitesses de transfert parties aériennes - racines et des vitesses de croissance différentes d'un traitement à l'autre. Au bout de 24 heures d'obscurité, les glucides sont pratiquement épuisés dans les parties aériennes à $25^{\circ} \mathrm{C}$ et dans les racines dès $20^{\circ} \mathrm{C}$. 


\section{B. Relation entre l'activité respiratoire et les teneurs en différentes fractions glucidiques}

Les hexoses (fructose et glucose), le saccharose et l'amidon ne présentent pas le même type de relation avec l'activité respiratoire. Fructose et glucose se comportent de façon identique, c'est pourquoi ils ne sont pas différenciés (fig. 3). Si leur teneur décroît avec la respiration, elle reste relativement élevée après 41 heures d'obscurité à 10 et $15^{\circ} \mathrm{C}$. Le saccharose est présent dans tous les cas en quantités plus réduites que les hexoses (fig. 4). Pour chaque traitement température.

Respiration $\left(\mu \mathrm{gCO} 2 \cdot g \mathrm{MS}^{-1} \cdot \mathrm{s}^{-1}\right)$
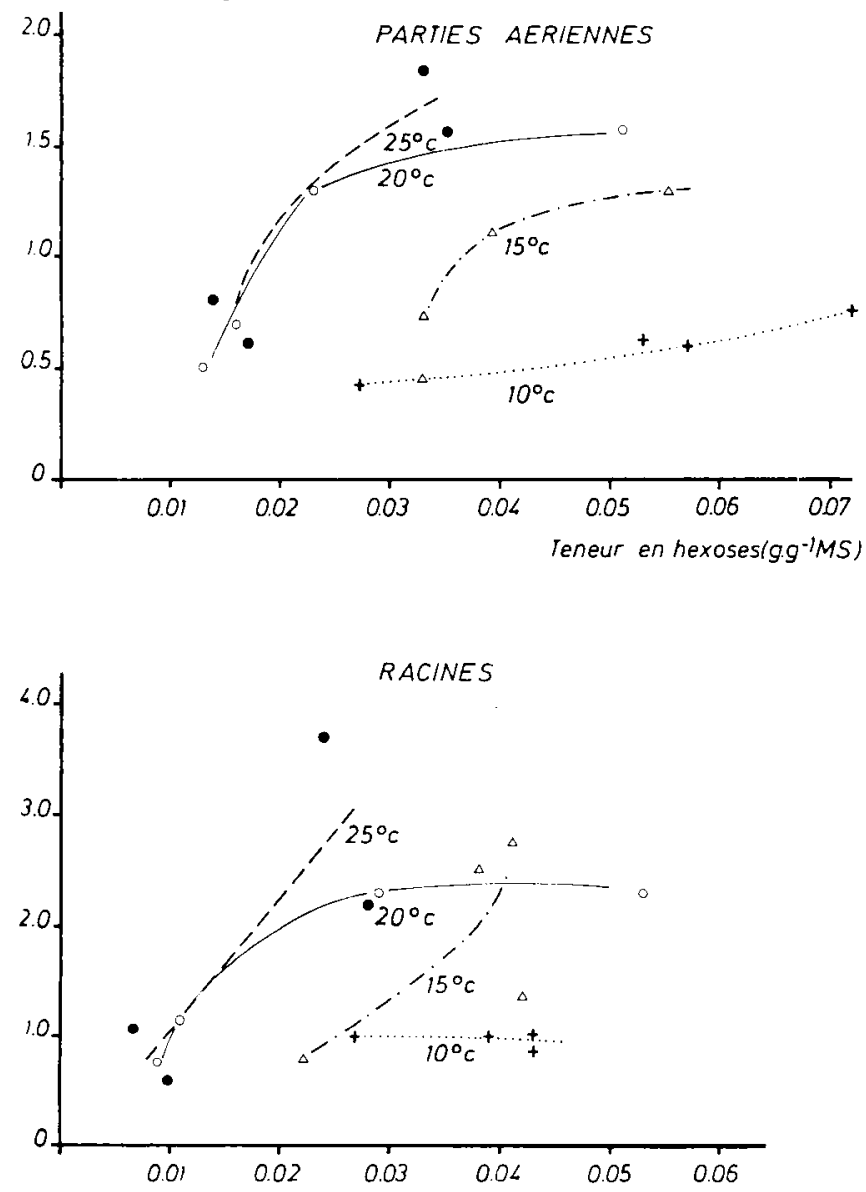

Figure 3

Relation entre activite respiratoire et teneur en hexoses (fructose, glucose) à différentes températures.

Respiration versus hexose (fructose, glucose) content at different temperatures.

l'activité respiratoire chute rapidement quand la teneur en saccharose passe en deçà d'une valeur seuil $(0,001$ à $\left.0,002 \mathrm{~g} \cdot \mathrm{g}^{1}\right)$. Cependant, quand la teneur en saccharose s'annule, l'activité respiratoire persiste, alimentée par de nouveaux substrats, vraisemblablement le fructose, le glucose ou l'amidon, non encore épuisés. Ceci est vrai aussi bien pour les parties aériennes que pour les racines. L'amidon est la fraction glucidique dominante dans les parties aériennes. Sa teneur décroît progressi-
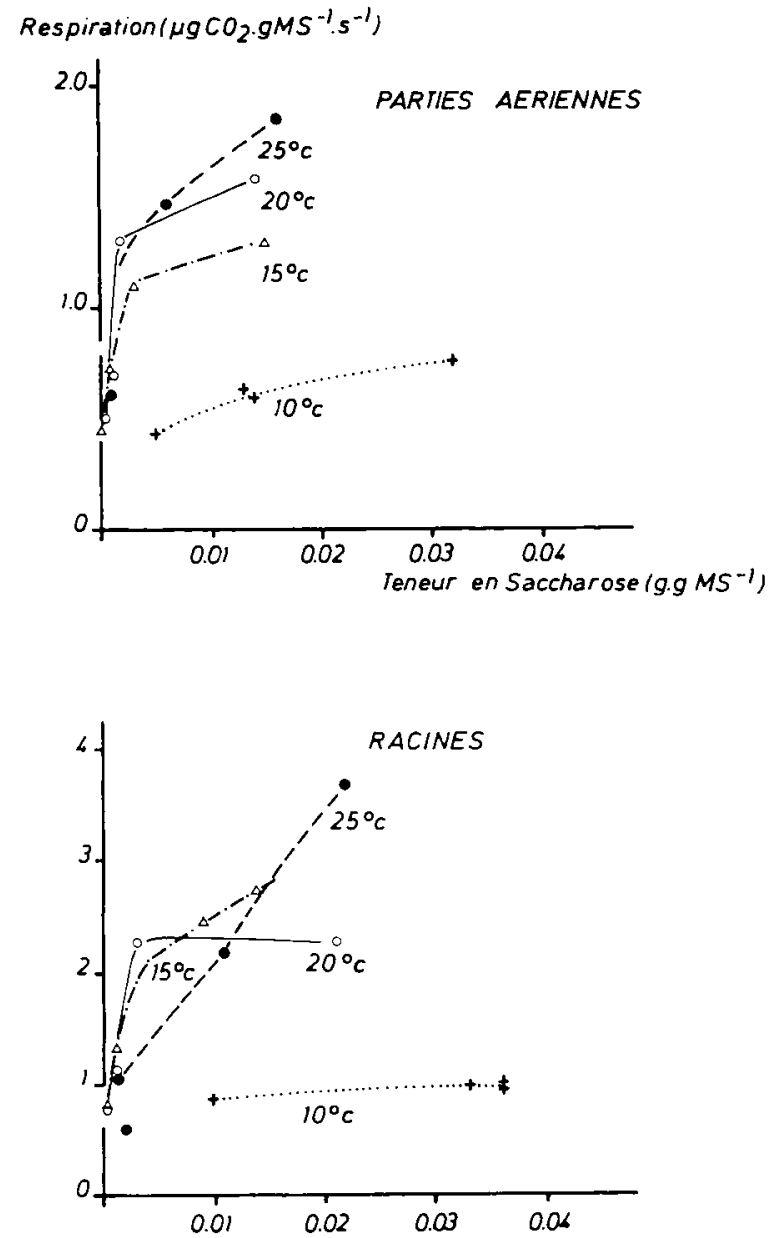

Figure 4

Relation entre activité respiratoire êt teneur en saccharose à différentes températures.

Respiration versus sucrose content at different temperatures.

vement (fig. 5). Cette décroissance s'accélère quand la teneur en saccharose devient très faible, à 15,20 et $25^{\circ} \mathrm{C}$.

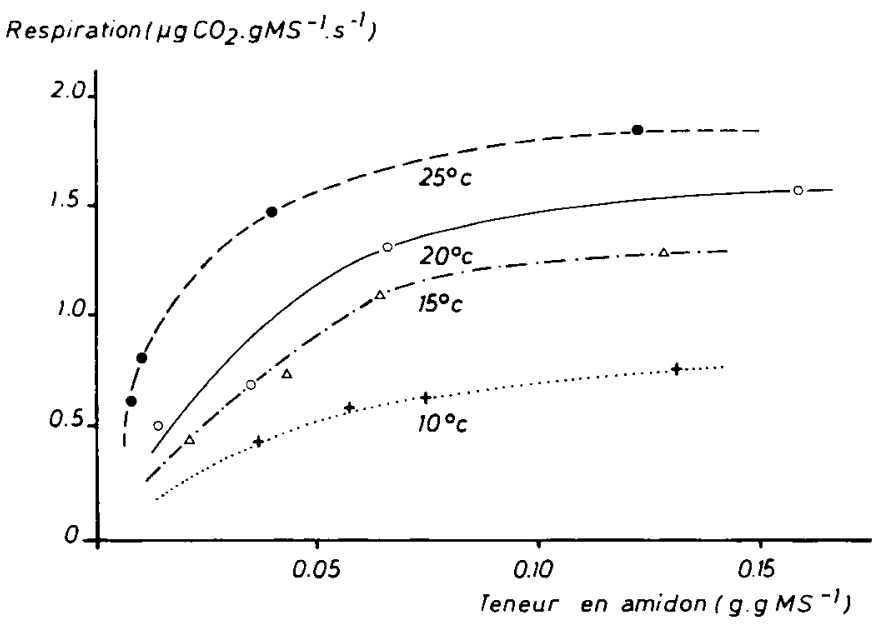

Figure 5

Relation entre activité respiratoire et teneur en amidon des parties aériennes à différentes températures.

Respiration versus starch content in shoots at different temperatures. 


\section{Relation entre respiration de la plante entière et teneur en glucides; effet de la température}

L'ensemble de ces résultats montre que les différentes fractions glucidiques peuvent être limitantes pour l'activité respiratoire, aussi bien dans les racines que dans les parties aériennes. Dans un objectif de modélisation, on peut, de la même façon mais à l'échelle de la plante entière, tracer les courbes de réponse de l'activité respiratoire à la teneur en glucides totaux (fig. 6). L'hypothèse d'une relation hyperbolique semble bien vérifiée. L'effet de la température, quant à lui, n'est pas indépendant de la quantité de substrat glucidique disponible. Très marqué aux fortes teneurs en glucides, il s'atténue progressivement.

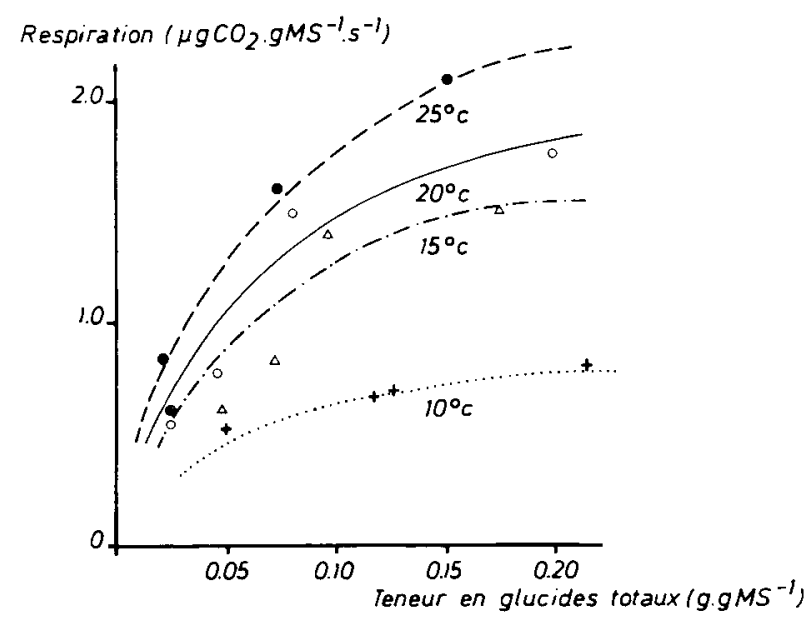

Figure 6

Relation entre activité respiratoire et teneur en glucides totaux de la plante entiere à différentes températures. Les courbes tracies sont les hyperboles theoriques.

Whole-plant respiration versus total carbohydrate content at different temperatures. The curves are the theoretical hyperbolae.

A $7{ }^{\circ} \mathrm{C}$, l'activité respiratoire est faible et varie très peu au cours du temps. Nous considérerons qu'elle est indépendante de la teneur en glucides. Pour ce traitement-là, $\mathrm{R}_{\max }$ (respiration maximale) est la moyenne des valeurs mesurées pendant 24 heures d'obscurité. Le faible nombre de points rend malaisée une détermination précise des paramètres de chaque hyperbole. Néanmoins, il apparaît que $\mathbf{R}_{\max }$ croît avec la température de façon exponentielle alors que $\mathrm{K}_{1 / 2}$ (teneur en glucides pour $\mathrm{R}=\mathrm{R}_{\max } / 2$, analogue au $\mathrm{K}_{\mathrm{m}}$ des réactions enzymatiques) semble dépendre peu de la température $\left(\mathrm{K}_{1 / 2} \approx 0,06 \mathrm{~g} \cdot \mathrm{g}^{-1}\right)$. Comme la plupart des plantes sensibles au froid ( chilling-sensitive plants »), la tomate voit son activité métabolique décroître brusquement entre 10 et $15^{\circ} \mathrm{C}$ (RAISON, 1980). Cela se traduit par une rupture de pente de la loi d'Arrhenius vers $11^{\circ} \mathrm{C}$ (fig. 7). La formulation suivante est proposée, avec des réserves liées au faible nombre de points disponibles:

$$
\begin{aligned}
& \mathrm{K}_{1 / 2}=0,06 \\
& \log \left(\mathrm{R}_{\text {max }}\right)=-3101 / \mathrm{T}+11,5 \text { pour } \mathrm{T} \geqslant 284 \mathrm{~K} \\
& \log \left(\mathrm{R}_{\max }\right)=-32540 / \mathrm{T}+115 \text { pour } \mathrm{T}<284 \mathrm{~K} .
\end{aligned}
$$

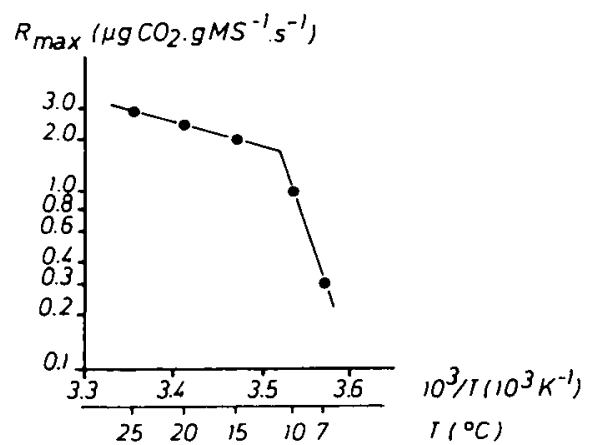

Figure 7

Relation entre respiration maximale et temperature.

Maximum respiration versus temperature

\section{DISCUSSION}

L'hypothèse avancée par THORNLEY (1972) selon laquelle il existe une relation michaelienne entre l'activité respiratoire de la plante entière et sa teneur en glucides semble convenir pour la tomate en phase végétative. Il y a interaction avec l'effet de la température; celui-ci porte essentiellement sur la respiration maximale.

L'activité respiratoire présente une relation de dépendance de forme identique vis-à-vis des différentes fractions glucidiques, avec quelques variations cependant. La diminution rapide d'activité quand la teneur en saccharose devient très faible pourrait s'interpréter comme étant la conséquence d'un arrêt des transferts. Les hexoses prendraient alors le relais du saccharose. A l'échelle de la plante entière, on fait abstraction de ces différences, ce qui revient à considérer la plante comme un amas de cellules indifférenciées ayant toutes accès de la même façon aux substrats respiratoires. Or il existe plusieurs compartiments glucidiques à l'intérieur même de la cellule (ROCHER \& Prioul, 1987). Le stockage de carbone dans le chloroplaste sous forme d'amidon le rend provisoirement inaccessible. Notons toutefois que l'activité amylasique n'est vraisemblablement pas limitante puisqu'elle est régulée par la teneur en saccharose dans le cytoplasme (STITT, 1984). Ce que traduit la diminution rapide de la teneur en amidon observée quand la teneur en saccharose devient très faible. De même, l'apparition d'organes d'accumulation immobilise plus ou moins définitivement une partie des assimilats.

Un autre point remarquable est la différence d'activité entre parties aérienne et souterraine. On peut invoquer le coût énergétique de l'absorption minérale. Par exemple, suivant leur vitesse de croissance, les racines de jeunes plants de maïs peuvent consacrer de 13 à 60 p. 100 de l'énergie respiratoire à l'absorption des anions (VEEN, 1980). Mais cela n'explique pas que les courbes de réponse à la teneur en glucides soient si différentes, bien que de forme identique. L'expression des résultats par unité de matière sèche est peut-être à mettre en cause. L'activité respiratoire mesurée devrait, en toute rigueur, être ramenée à la quantité d'enzymes actifs dans le métabolisme respiratoire.

Enfin, on peut s'interroger sur la permanence de la relation respiration -- teneur en glucides. Respiration et croissance sont étroitement liées. La première produit 
les chaînons carbonés, le pouvoir réducteur et l'énergie nécessaires à la deuxième qui fournit en retour NAD) et ADP, co-substrats indispensables au fonctionnement des différentes chaînes métaboliques de la respiration. Cette régulation étroite se traduit par la grande stabilité de la charge énergétique (PradeT \& RAymond, 1983). Or, les besoins énergétiques pour la croissance varient en fonction de l'état de croissance et de développement de la plante (Ruget et al., 1981 : STAHL \& MC Cree. 1988). PenNing de VRIEs et al. (1979) observent que la réponse de la respiration à la teneur en glucides est d'autant plus forte que les plantes sont jeunes, et donc que leur vitesse relative de croissance est plus grande. Mais, en exprimant leurs résultats uniquement par rapport à la masse des organes encore en croissance active, ils retrouvent des courbes de réponse identiques.
Ces quelques remarques limitent le domaine de validité des relations respiration - teneur en glucides température qui sont proposées ici au matériel végétal utilisé pour les établir. Pour simuler l'activité respiratoire dans des états de croissance et de développement différents, il faudrait prendre en compte les variations éventuelles des besoins en énergie respiratoire liés à la croissance, à l'entretien des structures de la plante et à l'absorption minérale (LAMBERS et al., 1983). Néanmoins, on peut penser que la forme de ces relations a une valeur générale. Leur introduction dans les modèles de bilan carboné permettrait en particulier la prise en compte des interactions entre les effets teneur en glucide et température sur l'activité respiratoire.

Reçl l' 15 décembre 1987. Accepté le 29 mars 1988

\section{RÉFÉRENCES BIBLIOGRAPHIQUES}

Ap Rees T., 1980. Assessment of the contributions of metabolic pathways to plant respiration, p. 1-29. In D. D. Davies, The biochemistry of plants, vol. 2, Academic Press, N.Y., $687 \mathrm{p}$

Azcón-Bieto J., Osmond C. B., 1983. Relationship betwecn photosynthesis and respiration. The effect of carbohydrate status on the rate of $\mathrm{CO}_{2}$ production by respiration in darkened and illuminated leaves. Plant Physiol., 71, 574-581.

Barker J., 1936. Analytical studies in plant respiration. VI. 3. The relation of the respiration to the concentration of sucrose. Proc. $R$. Soc. Londom Ser. B. biol. Sci., 119, 453-473.

Coggeshall B. M., Hodges H. F., 1980. The effect of carbohydrate concentration on the respiration rate of soybean. Crop Sci., 20, 86-90.

Frossard J. S., 1985a. L'éclairement du feuillage, facteur de régulation du rythme nycthéméral de la respiration des racines. Physioi. vég.. 23 (2), 163-173.

Frossard J. S., 1985h. Effet de la température des racines sur eur respiration et sur la croissance des plantules de 2 hybrides de mails. Agronomic, 5 (8), 719-725.

Gary C., 1988. Prise en compte des différentes sources d'erreurs et estimation de la précision dans la mesure des échanges de $\mathrm{CO}_{2}$ en système ouvert. Photosynthetica, 22 (1). 58-69.

Johnson I. R., Thornley J. H. M., 1985. Temperature dependence of plant and crop processes. Ann. Bot., 55, 1-24.

Lambers H., Szaniawski R. K., De Visser R., 1983. Respiration for growth, maintenance and ion uptake. An evaluation of concepts, methods, values and their signification. Phisiol. Plamt., 58, 556-563.

Longuenesse J. J., Conus G., Sarrouy C., 1982. Température nocturne et photosynthèse. 2. Une chambre d'assimilation climatisée pour la mesure des échanges gazeux de plantes cntières. Agronomic, 2 (8), $777-781$

Mc Cree K. J., 1982. Maintenance requirements of white clover at high and low growth rates. Crop $S c i ., 22,345-351$.
Penning De Vries F. W. T., Witlage J. M., Kremer D., 1979. Rates of respiration and of increase in structural dry matter in young wheat, rycgrass and maize plants in relation to temperature, to water stress and to their sugar content. Amm. Bot., 44, 595-609.

Pradet A., Raymond P., 1983. Adenine nucleotide ratios and adenylate energy charge in energy metabolism. Ann. Rev. Plant Physiol., 34, 199-224.

Raison J. K., 1980. Effect of low temperature on respiration, p. 613626. In D. D. Davies. The Biochemistry of Plants, vol. 2. Academic Press. N.Y., 687 p.

Rocher J. P., Prioul J. L., 1987. Compartmental analysis of assimilate export in a mature maize leaf. Plant Physiol. Biochem., 25 (5), 531-540. Ruget F., André M., Massimino J., 1981. Evolution de la respiration et croissance, au cours d'un cycle de végétation, de maïs cultivé en chambre de mesure. Physiol. vég., 19 (2), 277-299.

Saglio P. H., Pradet A., 1980. Soluble sugars, respiration, and encrgy charge during aging of excised maize root tips. Plant Phlysiol., 66, $516-519$.

Stahl R. S., Mc Cree K. J., 1988. Ontogenic changes in the respiration coefficients of grain sorghum. Crop Sci., 28, 111-113.

Stitt M., 1984. Degradation of starch in chloroplasts : a buffer to sucrose metabolism, p. 205-229. In D. H. Lewis, Storage carbohydrates in vascular plants. distribution, phrsiology and metabolism, S.E.B. seminar series ${ }^{\circ} 19$, Cambridge University Press, Cambridge, $284 \mathrm{p}$. Sweeley C. C., Bentley R., Makita M., Wells W. W., 1963. Gas-liquid chromatography of trimethylsilyl derivates of sugars and related substances. J. amer. chem. Soc., 85, 2497-2507.

Thornley J. H. M., 1972. A model to describe the partitioning of photosynthate during vegelative plant growth. Amn. Bot., 36, 419-430. Veen B. W., 1980. Energy cost of ion transport. p. 187-195. In D. W. Rains et al. Genetic cngineering of osmoregulation. Impact on plant productivity for food, chemical and energy. Plenum Press, N.Y.

Yemm E. W., 1965. The respiration of plants and their organs, p. 231-310. In F. C. Steward. Plant physiology', volume IV A. Academic Press, N.Y. 